\title{
X-ray spectral evolution of high redshift quasars
}

\section{Citation}

Bechtold, Jill, Martin Elvis, Fabrizio Fiore, Olga Kuhn, Roc M. Cutri, Jonathan C. McDowell, Marcia Rieke, Aneta Siemiginowska, and Belinda J. Wilkes. 1994. "X-Ray Spectral Evolution of High Redshift Quasars." The Astronomical Journal 108 (September): 759. doi:10.1086/117111.

\section{Published Version}

doi:10.1086/117111

\section{Permanent link}

http://nrs.harvard.edu/urn-3:HUL.InstRepos:30212145

\section{Terms of Use}

This article was downloaded from Harvard University's DASH repository, and is made available under the terms and conditions applicable to Other Posted Material, as set forth at http:// nrs.harvard.edu/urn-3:HUL.InstRepos:dash.current.terms-of-use\#LAA

\section{Share Your Story}

The Harvard community has made this article openly available.

Please share how this access benefits you. Submit a story.

Accessibility 


\title{
X-RAY SPECTRAL EVOLUTION OF HIGH REDSHIFT QUASARS
}

\author{
JILL BECHTOLD \\ Steward Observatory, University of Arizona, Tucson, Arizona 85721 \\ Electronic mail: jbechtold@as.arizona.edu \\ MARTIN Elvis, FABrizio Fiore, AND Olga KuHN \\ Harvard-Smithsonian Center for Astrophysics, 60 Garden Street, Cambridge, Massachusetts 02138 \\ Electronic mail: elvis@cfa.harvard.edu \\ ROC M. CUTRI \\ Steward Observatory, University of Arizona, Tucson, Arizona 85721 \\ Electronic mail: rcutri@as.arizona.edu \\ JONATHAN C. MCDOWELL \\ Harvard-Smithsonian Center for Astrophysics, 60 Garden Street, Cambridge, Massachusetts 02138 \\ Electronic mail: mcdowell@as.arizona.edu \\ MARCIA RIEKE \\ Steward Observatory, University of Arizona, Tucson, Arizona 85721 \\ Electronic mail: mrieke@as.arizona.edu \\ ANETA SiemiginowsKa AND BELINDA J. WiLKES \\ Harvard-Smithsonian Center for Astrophysics, 60 Garden Street, Cambridge, Massachusetts 02138 \\ Electronic mail: aneta@miles.harvard.edu \\ Received 1994 February 14; revised 1994 April 26
}

\begin{abstract}
At $z \approx 3$, the $\mathrm{x}$-ray spectra of radio-loud and radio-quiet quasars are different. High-redshift radio-quiet quasars either have large absorbing columns, $N_{\mathrm{H}}$, and steeper power law spectral indices, $\alpha_{E}$, than low redshift quasars, or no absorption and similar $\alpha_{E}$ 's. In contrast, the radio-loud quasars at high redshift have substantial absorption and similar $\alpha_{E}$ 's to low redshift quasars. Implications for the interpretation of the evolution of the luminosity function of quasars are discussed. If the absorption arises outside the central engine for both radio-loud and radio-quiet quasars, then radio-quiet quasars differ from the radio-loud quasars in that their emitted power law spectrum has evolved with redshift. We argue that this favors models where quasars are numerous and short-lived, rather than rare and long-lived.
\end{abstract}

\section{INTRODUCTION}

A basic characteristic of quasars is the scale-free nature of their emission. From the lowest luminosity AGN (e.g., M81) to the highest redshift, most luminous quasars, the quasar continuum and emission lines scale almost linearly with luminosity, regardless of redshift (e.g., Netzer 1990; Blandford 1990 , and references therein). This is true within both the radio-loud and the radio-quiet classes of quasar. There are small deviations from this pattern, e.g., the Baldwin effect (Baldwin 1977), and the slowly decreasing x-ray loudness for higher luminosity quasars (Avni \& Tananbaum 1986; Worrall et al. 1987). Even these, however, are continuous changes in the sense that there is no characteristic redshift or luminosity at which they occur.

By contrast, evolution studies show that quasars evolve strongly with redshift, and that $z \sim 2$ is a characteristic redshift for the quasar population. The break point, $L^{*}$, of their luminosity function shifts to higher luminosities by a factor of 40-50 between $z=0.1$ and $z=2$ (Boyle et al. 1987). There are two extreme possibilities for the physical evolution that causes the observed population evolution: individual objects are long-lived or short-lived. In the long-lived case, quasars are intrinsically rare, their present-day remnants have very high masses from the integrated accretion of mass over a Hubble time, and today they should emit at less than $1 \%$ of the Eddington luminosity, $L_{\text {Edd }}$ (Cavaliere \& Padovani 1988). In the alternative short-lived case, quasars are common, so that most large galaxies had one at one time, but each is active only for a short time $\left(\sim 10^{8} \mathrm{yr}\right)$. In order not to require too much mass tied up in black holes, these quasars must be accreting close to their Eddington limits while they are active (Small \& Blandford 1992; Chokshi \& Turner 1992).

Physical evolution models could be more easily distinguished if there were characteristic luminosities or redshifts at which the properties of individual quasars changed. Recent results presented by Elvis et al. (1994) and Bechtold et al. (1994) suggest that the continuum spectral energy distributions of quasars are in fact changing at $z \sim 2$. They find:

(1) Radio-loud quasars become strongly x-ray absorbed 
between $z=0.5$ and $z=3$, whereas radio-quiet quasars probably do not.

(2) Steep spectrum radio-loud quasars do not continue to follow the correlation of $\alpha_{\mathrm{ox}}$ increasing with $L_{\mathrm{opt}}$ at high redshift; they are too $\mathrm{x}$-ray bright. Radio-quiet quasars instead continue to follow the correlation of $\alpha_{\text {ox }}$ increasing with increasing $L_{\text {opt }}$ seen at lower redshift.

These observations suggest that breaks in the scaling laws for quasars are beginning to appear which may be related to the evolution of the quasar population as a whole. In this paper we review the data supporting these claims, and then discuss their potential value for understanding quasar emission mechanisms.

\section{X-RAY SPECTRA AND HARDNESS RATIOS}

Obtaining x-ray spectra of high redshift quasars has become feasible with the ROSAT (Trümper 1983) Position Sensitive Proportional Counter (PSPC, Pfefferman et al. 1987). Elvis et al. (1994, hereafter referred to as Paper I) presented $\mathrm{X}$-ray spectra of several radio-loud high-redshift quasars. Bechtold et al. (1994, hereafter referred to as Paper II) derived hardness ratios for a dozen $z \sim 3$ quasars, six of them radio-quiet. Although the small number of counts in these detections render their hardness ratios ambiguous, they nevertheless add significantly to our knowledge of quasar spectra at high redshift.

Since Paper II was completed, other PSPC data on high redshift quasars have been published (Fink \& Briel 1993; Henry et al. 1994; Molthagen et al. 1994; Band et al. 1994). This allows us to expand on the results given in Paper II. In Table 1, we list the X-ray results derived in Paper I and Paper II. We also give the results for OQ 172, which we reanalyzed in order to ensure uniformity of treatment, since the PSPC data have now become public. The $z=4.5$ quasar $0953+47$ observed by Molthagen et al. (1994) was not detected in the PSPC, but we include their limit for $\alpha_{\text {ox }}$ below.

The PSPC hardness ratio, defined as

$$
R=(H-S) /(H+S),
$$

is given in Table 1 for each quasar. Here $H$ is the number of counts in a hard band defined as PI channels $41-245$, or 0.41 to $2.48 \mathrm{keV}$ in the observed frame; $S$ is the number of counts in a soft band defined as PI channels 11-40, or observed energies of 0.11 to $0.40 \mathrm{keV}$. Note that for an object at $z=3$, these channels correspond to $1.64-9.92 \mathrm{keV}$ and $0.44-1.60$ $\mathrm{keV}$, respectively. In addition to the high-redshift quasars, we include four intermediate redshift, radio-quiet quasars with $1.5<z<2.2$ found in the PSPC field of Q0130-403 (see Paper II; Hoag \& Smith 1977; Osmer 1977).

Figure 1 is a plot of $R$ for the quasars in our sample. Clearly, there is a systematic difference between the radioloud and radio-quiet quasars. Omitting the three cases where the galactic column density is larger than $7 \times 10^{20}$ atoms $\mathrm{cm}^{-2}$ (implying transmission of only $10 \%$ at the carbon edge and hence a large effect on $R$ ), the mean $R$ for radio-quiet quasars is $-0.018 \pm 0.110$, while for the radioloud quasars the mean $R$ is $0.78 \pm 0.03$, a $7.2 \sigma$ difference.
TABLE 1. X-ray and hardness ratios.

\begin{tabular}{|c|c|c|c|c|c|}
\hline quasar & $\mathrm{z}_{e m}$ & $\mathrm{~N}_{H G a l}^{a}$ & $\mathrm{R}^{b}$ & $\begin{array}{c}\text { Case A } \\
\alpha_{E}\end{array}$ & $\begin{array}{c}\text { Case B } \\
\alpha_{E}\end{array}$ \\
\hline $0000-263$ & 4.11 & 1.60 & $-0.065 \pm 0.183$ & $1.30_{-0.23}^{+0.23 c}$ & $2.20_{-0.30}^{+0.27^{d}}$ \\
\hline $0130-403$ & 3.03 & 2.30 & $-0.170 \pm 0.372$ & $1.69_{-0.60}^{+0.71 c}$ & $2.30_{-0.55}^{+0.80^{d}}$ \\
\hline 0207-398 & 2.81 & 1.53 & $0.128 \pm 0.216$ & $0.90_{-0.36}^{+0.34 c}$ & $1.60_{-0.40}^{+0.30^{d}}$ \\
\hline $1107+481$ & 2.96 & 1.40 & $0.066 \pm 0.257$ & $0.93_{-0.39}^{+0.39 c}$ & $1.61_{-0.48}^{+0.39^{d}}$ \\
\hline $1208+101$ & 3.82 & 1.70 & $-0.249 \pm 0.346$ & $1.54_{-0.53}^{+0.73 c}$ & $2.05_{-0.60}^{+0.70^{d}}$ \\
\hline $1946+768$ & 3.02 & 7.50 & $0.935 \pm 0.157$ & $1.00_{-0.32}^{+0.28 c}$ & $1.23_{-0.39}^{+0.37 d}$ \\
\hline $0130-401$ & 1.73 & 2.30 & $-0.154 \pm 0.269$ & $1.66_{-0.44}^{+0.51^{c}}$ & $3.88_{-0.60}^{+0.60^{e}}$ \\
\hline $0130-404$ & 2.16 & 2.30 & $0.081 \pm 0.465$ & $1.27_{-0.80}^{+0.79 c}$ & $2.88_{-0.99}^{+0.93 e}$ \\
\hline $0131-401 \mathrm{~A}$ & 1.83 & 2.30 & $-0.249 \pm 0.204$ & $1.83_{-0.36}^{+0.38 c}$ & $3.93_{-0.43}^{+0.47 e}$ \\
\hline 0131-401C & 1.65 & 2.30 & $0.129 \pm 0.339$ & $1.20_{-0.59}^{+0.53^{c}}$ & $3.40_{-0.70}^{+0.67^{e}}$ \\
\hline $0014+813$ & 3.38 & 14.4 & $0.962 \pm 0.077$ & $0.82_{-0.19}^{+0.19^{c}}$ & $0.97_{-0.20}^{+0.20^{d}}$ \\
\hline 0420-388 & 3.12 & 1.91 & $0.406 \pm 0.074$ & $0.73_{-0.10}^{+0.10^{c}}$ & $1.24_{-0.48}^{+0.48 d}$ \\
\hline $0438-436$ & 2.85 & 1.50 & $0.903 \pm 0.071$ & $0.61_{-0.22}^{+0.22 f}$ & $0.61_{-0.22}^{+0.22 f}$ \\
\hline $0636+680$ & 3.17 & 5.70 & $1.140 \pm 0.276^{g}$ & $-0.24_{-0.44}^{+0.50 c}$ & $0.04_{-0.54}^{+0.50^{d}}$ \\
\hline $1442+101$ & 3.54 & 1.73 & $0.480 \pm 0.067$ & $0.45_{-0.09}^{+0.09 c}$ & $0.45_{-0.10}^{+0.10^{f}}$ \\
\hline $1745+624$ & 3.87 & 3.40 & -- & $0.30_{-0.60}^{+0.60 f}$ & $0.30_{-0.60}^{+0.60 f}$ \\
\hline $2000-330$ & 3.78 & 7.50 & $0.721 \pm 0.242$ & $1.61_{-0.92}^{+0.71^{c}}$ & $1.49_{-0.57}^{+0.53 d}$ \\
\hline 2126-158 & 3.27 & 4.85 & $0.963 \pm 0.041$ & $0.52_{-0.21}^{+0.25 f}$ & $0.52_{-0.21}^{+0.25 f}$ \\
\hline
\end{tabular}

Notes to TABLE 1

${ }^{\mathrm{a}}$ In units of $10^{20}$ atoms $\mathrm{cm}^{-2}$. ${ }^{\mathrm{b}}$ Hardness ratio, $R=H-S / H+S$; for 1745 +624 the PSPC data are not available. ${ }^{c} N_{\mathrm{H}}$ fixed to the galactic value. ${ }^{\mathrm{d}}$ Additional $N_{\mathrm{H}}$ at $z=z_{\mathrm{em}}$ fixed to $10^{22}$ atoms $\mathrm{cm}^{-2}$. ${ }^{\mathrm{e}}$ Additional $N_{\mathrm{H}}$ at $z=z_{\mathrm{em}}$ fixed to $5 \times 10^{21}$ atoms $\mathrm{cm}^{-2}$. ${ }^{\mathrm{f}}$ Best fit additional $N_{\mathrm{H}}$ at $z=z_{\mathrm{em}}$. ${ }^{\mathrm{g}}$ Formally, $R>1$ since $S=-4.8 \pm 6.5$ after background subtraction. Only values of $R<1$ and $S>0$ are physical, however.

The dashed and dotted lines in Fig. 1 represent the expectation for radio-loud and radio-quiet quasars, respectively, using the mean spectral index found at low redshift by Ginga (0.71 for radio-loud and 1.03 for radio-quiet, Williams et al. 1992 ) and the minimum and maximum galactic $N_{\mathrm{H}}$ values in our samples $\left(1.4-2.3 \times 10^{20}\right.$ atoms $\mathrm{cm}^{-2}$ for the radio-quiet quasars and $1.5-5.7 \times 10^{20}$ atoms $\mathrm{cm}^{-2}$ for the radio-loud quasars). The radio-quiet quasars have $R$ compatible with the low redshift predictions, while at least some of the radioloud quasars have $R$ definitely greater than predicted. We compare our results to the Ginga data for low redshift quasars, since for $z \sim 3$ the PSPC bandpass corresponds to rest frame energies of $\sim 1-10 \mathrm{keV}$, similar to the Ginga bandpass for low redshift objects. For the $z \sim 2$ quasars, the PSPC bandpass corresponds to $\sim 0.6-7 \mathrm{keV}$, between the harder X-rays observed by Ginga $(2-10 \mathrm{keV})$ and the softer x-rays observed by the IPC $(0.1-3.5 \mathrm{keV})$ for low redshift objects. However, this energy difference is probably not important for these comparisons since the Ginga and IPC measured slopes are similar (Fiore et al. 1994).

The difference in $R$ between low and high redshift radioloud quasars, can be interpreted in different ways. The two 


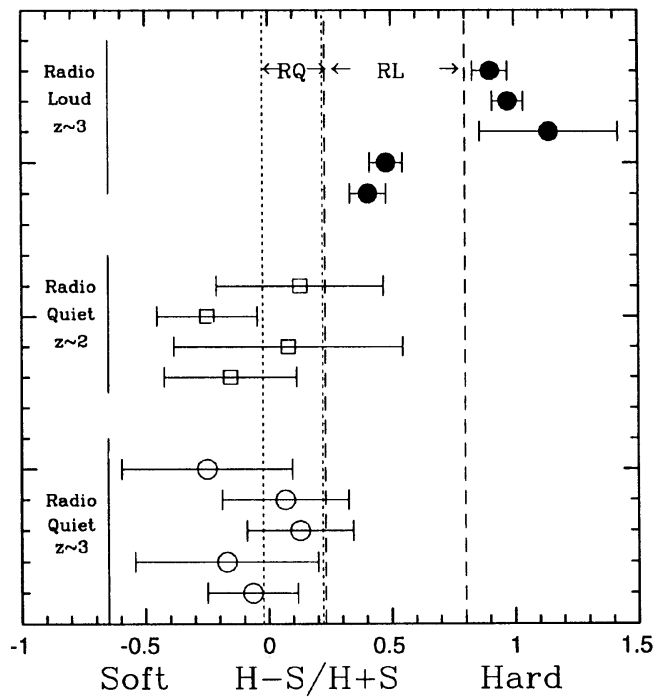

Fig. 1. The ROSAT PSPC hardness ratio for a sample of high-redshift quasars. Open symbols identify radio-quiet quasars, filled symbols identify radio-loud quasars: circles correspond to $2.8<z<4.1$ quasars; squares corresponds to $1.6<z<2.2$ quasars. The dotted and dashed lines show the range of hardness ratio expected from the low $z$ Ginga observations. $H$-number of counts in the hard PSPC band $(0.41-2.48 \mathrm{keV}) ; S$-number of counts in the soft PSPC band $(0.11-0.40 \mathrm{keV})$.

most simple and extreme possibilities are (A) a difference in emitted power law index, in the sense that the high-redshift quasars are flatter than the low redshift ones, or (B) a difference in absorbing column density, in the sense that the high $z$ quasars have a larger absorbing column than at low $z$. Of course intermediate situations, where both the emitted power law index and the absorption properties of high $z$ quasars are different, are also possible. In Table 1 , we list the spectral index $\alpha_{E}$ obtained for both cases (A) and (B) using the hardness ratio diagrams discussed in Paper II, for the radio-quiet quasars Q0130-403, Q0207-398, Q1107+481 and Q1208 +101 , and for the intermediate redshift radio-quiet quasars in the field of Q0130-403. For the other quasars, the $\alpha_{E}$ obtained from normal spectral fitting is given (Paper I).

For case (A), $N_{\mathrm{H}}$ was assumed to be equal to the galactic $N_{\mathrm{H} \text { I }}$ value in all the objects for which this assumption is statistically consistent with the observed spectrum (i.e., all radio-quiet quasars, and all the radio-loud quasars with the exception of PKS 0438-436, PKS 2126-158, see Papers I, II). For case (B), an absorber with the best fit $N_{\mathrm{H}}$ at $z=z_{\text {em }}$ (as given in Paper I) was assumed for PKS 0438-436 and PKS 2126-158. Otherwise, a column of $N_{\mathrm{H}}=10^{22}$ atoms $\mathrm{cm}^{-2}$ at $z_{\mathrm{em}}$ was assumed in addition to the galactic $N_{\mathrm{H}}$. In these cases, the statistics of the data are not good enough to constrain the intrinsic $N_{\mathrm{H}}$ (this includes all the radio-quiet quasars and the radio-loud quasars Q0014 +813 , Q0420-388, Q0636+680, and PKS 2000-330). In both cases the absorbers were assumed to have cross sections given by Morrison \& McCammon (1983) with solar metal abundance. We note that two of the eight radio-loud quasars, Q1745+624 (Fink \& Briel 1993) and OQ 172, do not show evidence for intrinsic absorption. The limit for intrinsic absorption quoted by Fink \& Briel for the first object is $2 \times 10^{20}$

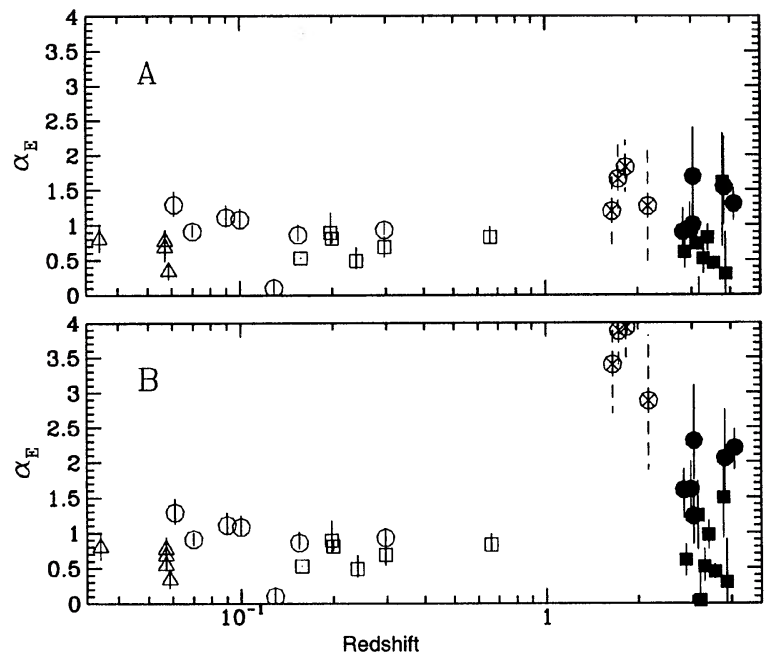

FIG. 2. X-ray energy spectral index in the $1-10 \mathrm{keV}$ emitted frame vs redshift when assuming: (a) $N_{\mathrm{H}}$ at $z=z_{\mathrm{em}}$ of $10^{22}$ atoms $\mathrm{cm}^{-2}$ for $z \sim 3$ quasars and $5 \times 10^{21}$ atoms $\mathrm{cm}^{-2}$ for $z \sim 2$ quasars; (b) $N_{\mathrm{H}}$ fixed at the galactic value. Squares $=$ radio-loud quasars, circles $=$ radio-quiet quasars: triangles $=$ radio galaxies; filled symbols $=$ ROSAT PSPC $z \sim 3$ quasars; open circles with crosses $=$ quasars with $1.6<z<2.2$. Open symbols and triangles $=$ Ging $a$ results.

atoms $\mathrm{cm}^{-2}$, while for the second object we found a $99 \%$ limit of $0.5 \times 10^{22}$ atoms $\mathrm{cm}^{-2}$.

\section{X-RAY SPECTRAL EVOLUTION}

How does $\alpha_{E}$ evolve with redshift?

Figures 2 and 3 show $\alpha_{E}$ as a function of redshift (Fig. 2) and luminosity (Fig. 3) for the objects in this sample,

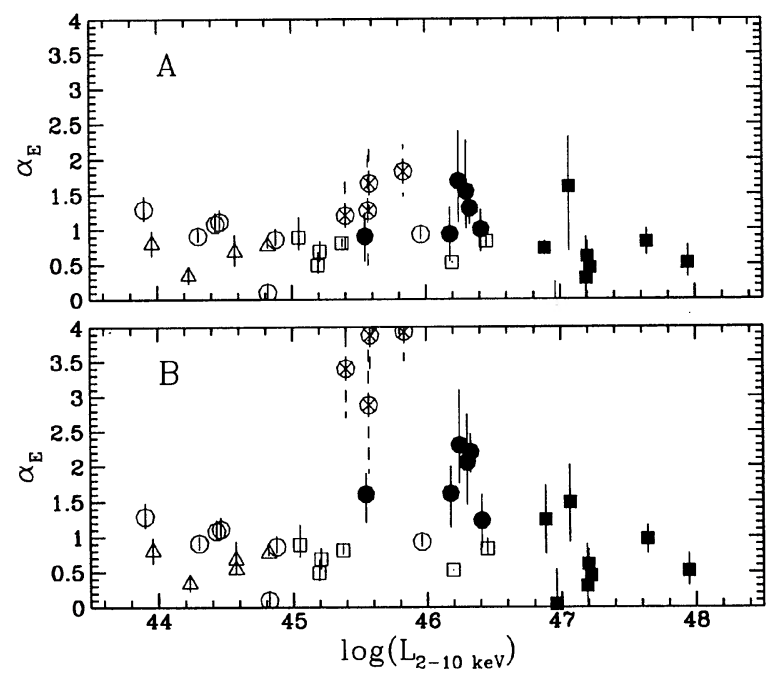

Fig. 3. X-ray energy spectral index in the $1-10 \mathrm{keV}$ emitted frame vs $2-10$ keV luminosity when assuming: (a) $N_{\mathrm{H}}$ at $z=z_{\mathrm{em}}$ of $10^{22}$ atoms $\mathrm{cm}^{-2}$ for $z \sim 3$ quasars and $5 \times 10^{21}$ atoms cm $\mathrm{cm}^{-2}$ for $z \sim 2$ quasars; (b) $N_{\mathrm{H}}$ fixed at the galactic value. Squares=radio-loud quasars, circles =radio-quiet quasars: triangles $=$ radio galaxies; filled symbols $=R O S A T$ PSPC $z \sim 3$ quasars; open circles with crosses $=$ quasars with $1.6<z<2.2$. Open symbols and triangles $=$ Ging $a$ results. 
and for a low redshift comparison, quasars observed with Ginga. Note that the sizes of the low redshift and high redshift samples are similar. Figures 2 and 3 are updated versions of those in Paper I. Note that the PSPC spectral index covers $0.4-9.4 \mathrm{keV}$ for the lowest redshift object $(z=2.81)$, and $0.6-12.7 \mathrm{keV}$ for the highest redshift object $(z=4.11)$. This introduces some ambiguity, although not a large one if the Ginga low $z$ spectra are a reliable guide (Williams et al. 1992). We note also that the high redshift quasars in our sample are also the most luminous, so it is hard to distinguish whether the $\mathrm{x}$-ray spectral properties depend primarily on $z$ or $L_{\mathrm{x}}$. In the remainder of the discussion we consider the dependence of $\alpha_{E}$ on $z$, for case (A) and case (B).

Case (A): Only radio-loud high $z$ quasars are absorbed [Figs. 2(a),3(a)]. In this case, the difference in slope between radio-loud and radio-quiet quasars which was found at low redshifts (Wilkes \& Elvis 1987; Williams et al. 1992) persists at $z \sim 3$, radio-quiet quasars having steeper slopes. The weighted mean spectral slope of the radio-quiet quasars is $1.15 \pm 0.14$ (the error represents the error on the mean), consistent with the $\left\langle\alpha_{E}\right\rangle=1.03$, for a sample of six low redshift radio-quiet quasars (Williams et al. 1992; excluding the peculiar object PG 1416-129, which has a best fit $2-10 \mathrm{keV}$ slope of only 0.1 ). The constraint on any change of $\alpha_{E}$ with $z$ is no more than 0.3 (99\% confidence). For the radio-loud quasars the weighted mean spectral slope is $0.59 \pm 0.06$, comparable with the $\left\langle\alpha_{E}\right\rangle=0.71$ for a sample of five low-redshift radio-loud quasars (Williams et al. 1992).

In case (A) then radio-quiet quasars show no change in either emitted slope or absorption out to $z=4$, while radioloud quasars commonly exhibit strong x-ray absorption at $z=3$, with no change in intrinsic slope. This immediately implies that the absorption in radio-loud $z \sim 3$ quasars (Paper I) is primarily intrinsic to the quasars, since intervening absorbers would be found independently of the radio properties of the quasar. (The number of objects is still small, however.)

The slope obtained for the intermediate redshift quasars, assuming galactic $N_{\mathrm{H}}$ (case A), is steeper than the IPC and Ginga slopes and is similar to those found in the PSPC spectra of low-redshift radio-quiet quasars (Walter \& Fink 1993; Laor et al. 1994; Fiore et al. 1994), despite the significantly lower energy range covered by the PSPC. In this case then the major evolution in X-ray slope occurs between $z \sim 0$ and $z \sim 2$, with little change thereafter up to $z \sim 3$. This behavior would be similar to the evolution of the quasar luminosity function (e.g., Boyle et al. 1993).

Case (B): All high $z$ quasars are absorbed [Figs. $2(b), 3(b)]$. No matter where the absorbers are located, the $\mathrm{x}$-ray hardness ratios of the radio-quiet quasars imply $\alpha_{E}$ 's which are steeper by $\sim 0.7$ than the no absorption case (see Table 1).

Figures 2(b) and 3(b) shows $\alpha_{E}$ as a function of x-ray luminosity and redshift for case (B). The mean $\alpha_{E}$ for the high-redshift radio-quiet quasars is $\left\langle\alpha_{E}\right\rangle=1.82 \pm 0.17$. This is significantly steeper than $\left\langle\alpha_{E}\right\rangle=1.03$ for the similar lowredshift quasars observed with Ginga. The two distributions of low and high redshift radio-quiet quasars spectral indices are different at the $97 \%$ confidence level (using the Kolmogorov-Smirnov test). The high-redshift radio-quiet

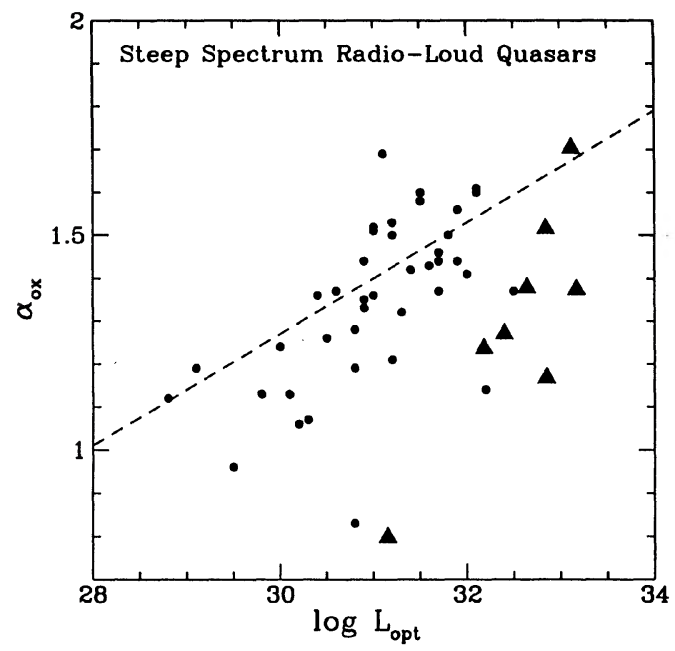

FIG. 4. $\alpha_{\text {ox }}$ vs $\log L_{\text {opt }}$ in erg s ${ }^{-1}$, for steep spectrum, radio-loud quasars. Filled circles are quasars detected with the Einstein IPC (Wilkes et al. 1994; Paper II) with $\alpha_{\text {radio }}>0.8$. Triangles are $z \sim 3$ quasars reported in Papers I and II. The dashed line is the predicted relation for steep spectrum radio sources from Worrall et al. (1987). The high redshift quasars are more $\mathrm{x}$-ray bright than the low redshift relation predicts.

mean slope is also different from that of high-redshift radioloud quasars which demonstrably show absorption $\left(\left\langle\alpha_{E}\right\rangle=0.52 \pm 0.16\right)$, and that of all high-redshift radio-loud quasars $\left(\left\langle\alpha_{E}\right\rangle=0.57 \pm 0.08\right)$.

The intermediate redshift quasars would have steeper slopes $\alpha_{E} \sim 4$ on this assumption. They would then be steeper at $z \sim 1.5$ than at either $z \sim 0$ or $z \sim 3$. This seems unlikely, but is not ruled out. We note that the path length for absorption, $X$ (Bahcall \& Peebles 1969), is half as large at the mean redshift of the quasars in the field of Q0130-403 $(z=1.84)$ than of the absorbed quasars in Paper I, so the chance of intervening absorbers is similarly reduced.

In summary, in either case (A) or (B), the $\mathrm{x}$-ray colors and spectra suggest that radio-quiet and radio-loud objects have evolved differently since $z \approx 3$. If the radio-quiet objects have, on average, as much absorption as the radio loud quasars, their power laws must be steeper than their low redshift counterparts, indicating evolution in the emission spectrum with redshift. If only radio-loud quasars have absorption, then the high-redshift radio-loud objects differ from both their low $z$ counterparts and from the radio-quiet quasars in that they have substantial absorbing material.

$$
\text { 4. X-RAY LOUDNESS }\left(\alpha_{\mathrm{ox}}\right) \text { VERSUS } L_{\mathrm{opt}}
$$

The plots of x-ray loudness, $\alpha_{\mathrm{ox}}$ (e.g., Tananbaum et al. 1979), versus $L_{\text {opt }}$ in Figs. 4 and 5 are updated versions of that in Paper II. The triangles mark the high redshift quasars from Paper II, Band et al. (1994), and Molthagen et al. (1994); the small circles show the quasars from the Einstein IPC survey (Wilkes et al. 1994) which are predominantly at low $(z<1)$ redshift. Figure 5 shows only radio-quiet quasars, while Fig. 4 contains only radio-loud quasars. Moreover, we have restricted the radio-loud quasars to those in which beaming is not likely to be important, i.e., lobe-dominated or 


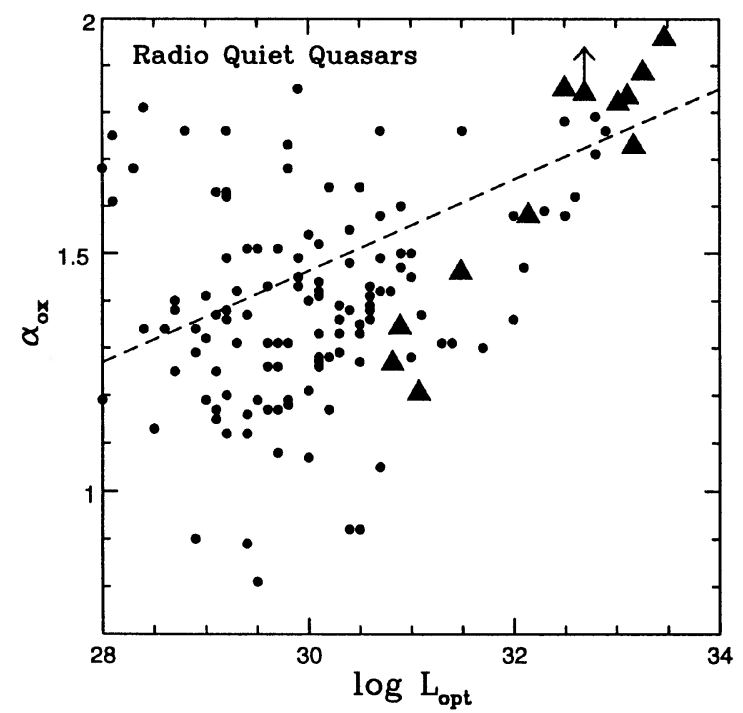

FIG. 5. $\alpha_{\text {ox }}$ vs $\log L_{\text {opt }}$ in erg s ${ }^{-1}$ for radio-quiet quasars. Filled circles are quasars detected with the Einstein IPC (Wilkes et al. 1994; Paper II). Triangles are radio-quiet quasars presented in Paper II. The dashed line is the predicted relation for radio-quiet quasars from Worrall et al. (1987). The high redshift quasars are consistent with the low redshift relation.

steep spectrum radio sources $\left(\alpha_{\text {radio }}>0.8\right)$. Beaming is not thought to be important in GPS sources and therefore in most of our high-redshift, radio-loud quasars.

The dashed lines show the predicted relations from Worrall et al. (1987) for each class of quasar. The high-redshift, radio-quiet quasars are consistent with the extrapolation from low redshift, and may even be slightly $\mathrm{x}$-ray quiet (cf. Pickering \& Impey 1994). In contrast, for steep-spectrum radioloud quasars, the high redshift quasars lie systematically below the prediction. They are too $\mathrm{x}$-ray loud for their optical luminosity. Note that despite the relatively small number of quasars detected in the IPC, the uncertainty in the predicted line is small on this scale.

While the range of observed $\alpha_{\text {ox }}$ of the high $z$ radio-loud quasars in Paper I ranges between 1.1 and 1.6, they are bunched toward small values of $\alpha_{\text {ox }}$ (Fig. 5). There are three cases with $\alpha_{\text {ox }}<1.3$ and only one with $\alpha_{\text {ox }} \sim 1.6$. This is S4 $0636+680$ which interestingly is also the most radio-quiet quasar in the radio-loud sample. A $\Delta \alpha_{\text {ox }}$ of 0.3 corresponds to a factor $\sim 6$ in $\mathrm{x}$-ray to optical flux ratio, so that this is a large effect.

This break in the predictive power of the $\alpha_{\mathrm{ox}}$ vs $L_{\mathrm{opt}}$ relations indicates a characteristic luminosity at $\log L_{\text {opt }} \sim 32-33 \mathrm{erg} \mathrm{s}^{-1} \mathrm{~Hz}^{-1}$, for steep-spectrum radio-loud quasars.

\section{DISCUSSION}

One can use these observations to speculate on the physical nature of the evolution of quasars in different scenarios. As mentioned briefly in the introduction there are two extreme possibilities: individual quasars are long-lived or short-lived. In the long-lived case, quasars are intrinsically rare, their present day remnants have very high masses from the integrated accretion of mass over a Hubble time, and today they should emit at less than $1 \%$ of the Eddington luminosity, $L_{\text {Edd }}$ (Cavaliere \& Padovani 1988). Since there is no evidence for very large black hole masses in nearby Seyferts or ellipticals and since there are indications that the ratio of the luminosity to its Eddington value in nearby Seyferts and intermediate redshift quasars is between 0.01 and 0.1 (Wandel \& Mushotzky 1986; Padovani \& Rafanelli 1988; Chokshi \& Turner 1992; Small \& Blandford 1992), it seems unlikely that the evolution of the luminosity function could be explained by the gradual decline in luminosity of individual, rare objects. A further test for the long-lived option is the evolution of the quasar continuum, since as the quasar black holes grow larger and become more starved of accreting matter, the continuum they produce is likely to change in form.

Alternatively, in the short-lived case, quasars are common (so that most large galaxies had one at one time), but each is active only for a short time $\left(\sim 10^{8} \mathrm{yr}\right)$. In order not to require too much mass tied up in black holes, these quasars must be accreting close to their Eddington limits while they are active (Small \& Blandford 1992). Haehnelt \& Rees (1993) have discussed the formation of such black holes in the context of the hierarchical collapse of cold dark matter fluctuations. At high redshifts only the most massive fluctuations can collapse; as time goes on, and the universe expands, less overdense structures collapse. Thus, the black hole mass of a typical quasar should strongly decrease with redshift, rather than slowly increase by mass accretion. This gives rise to opposite expectations about the quasar continuum evolution compared to the long-lived model. Any systematic differences in the spectral energy distributions of high and low redshift quasars could therefore guide and constrain models for the evolution of the quasar luminosity function.

\section{$5.1 X$-ray Spectral Evolution}

In Sec. 2, we have shown that radio-loud and radio-quiet quasars at high redshift have different $\mathrm{x}$-ray colors, but whether this is the result of different intrinsic $\mathrm{x}$-ray power laws or different absorbing columns cannot be determined from the present data. We discuss the implications of both possibilities.

How does the $x$-ray spectral index depend on physical models of evolution? In the standard accreting massive black hole model, simple assumptions can give the sense of the dependence of $\alpha_{E}$ on the black hole mass and accretion rate.

Comptonization of soft photons by a population of thermal electron in a hot corona above a layer of cold reflecting matter has been proposed as the origin of the $2-10 \mathrm{keV}$ spectrum in radio-quiet quasars (Haardt \& Maraschi 1993). In this model sources powered by accretion onto large black holes $\left(\approx 10^{10} \mathscr{l}_{\odot}\right.$, such as those needed at the center of these luminous $z \sim 3$ quasars) result in $2-10 \mathrm{keV}$ spectra which are significantly steeper than those of sources with smaller black holes. This holds for a range of corona optical depths, and therefore luminosities (Haardt \& Maraschi 1993).

If the evolution of the quasar luminosity function results 
from the slow evolution of a few, long-lived quasars, then one expects that that the black hole masses increase with decreasing $z$, with $L / L_{\text {Edd }} \sim 1$ at $z=3$, and $L / L_{\text {Edd }} \ll 1$ at $z=0$. The Haardt \& Maraschi (1993) models would then predict that $\alpha_{E}$ would steepen with decreasing $z$. On the other hand, if quasars are numerous, short-lived, and result from collapsing CDM halos in the Haehnelt \& Rees (1993) picture, then a typical quasar black hole mass will decrease with $z$. Then the $\mathrm{X}$-ray spectra will be steeper with increasing $z$.

In case (B), where all quasars are absorbed, radio-quiet quasars are steeper at higher $z$. This is opposite to the expectations for rare, long-lived quasars, but in agreement with the short-lived quasars in the Haehnalt and Rees picture.

In this case, the lack of evolution of the emitted $x$-ray power law for the radio-loud quasars is also significant. It would imply that their $\mathrm{x}$-ray emission is not closely related to the black hole mass. In fact, at low redshift, radio-loud quasars are also more x-ray loud (i.e., have smaller $\alpha_{\mathrm{ox}}$ ) than radio-quiet quasars. This supports the notion that there is an "extra" component of the x-ray emission related to the radio source. We return to this in our discussion of $\alpha_{\text {ox }}$ vs $L_{\text {opt }}$ in the next section.

In case (A), where only high $z$ radio-loud quasars are absorbed, neither the radio-quiet nor radio-loud quasars have changed their $\mathrm{x}$-ray spectral indices with redshift. Then one is forced to conclude that the $\mathrm{x}$-ray emission is not related in a direct way to black hole mass for either radio-quiet or radio-loud quasars, or that the number of quasars studied is so small that only the most extreme members of the population, all with nearly the same black hole mass, have been sampled.

Case (A) has other strong implications. Since soft x-ray absorption appears at high redshift only for the radio-loud quasars then (1) quasar radio emission results from a different environment in the high-redshift objects; (2) a dust shrouded phase of quasar development may be associated with radio-emission and be short-lived, having the same fractional life as the ratio of radio-loud to radio-quiet quasars. Some support for the second hypothesis comes from the fact that about $50 \%$ of the high-redshift radio-loud quasars are GHz Peaked Spectrum (GPS) sources, while the fraction is much reduced at low redshift (O'Dea et al. 1991). In fact five of the eight radio-loud quasars in our sample are candidate GPS sources in the list of O'Dea et al. (1991; Q0420 -388, Q0636+680, OQ 172, PKS 2000-330, and PKS 2126-158). PKS $0438-436$ also has a radio spectrum that resembles that of GPS sources. GPS sources are unusually compact $(\sim 10$ milliarcsec, $\sim 100$ pc at $z=3$, Pearson \& Readhead 1984). This compactness could be evidence for a dense surrounding medium which confines the radio source. This hypothesis is discussed in more detail in Paper I.

\subsection{X-ray Loudness $\left(\alpha_{o x}\right)$}

The presence of a critical luminosity above which radioloud quasars have a smaller optical to $x$-ray luminosity ratio than the less luminous, low-redshift ones (Sec. 4) suggests that the $\mathrm{x}$-ray emission of these objects becomes dominated by some mechanism not directly connected with the accretion rate, or limited by the Eddington luminosity.

An oft-suggested mechanism of this kind is the electromagnetic extraction of the black hole rotational energy (Blandford \& Znajeck 1977), which would be converted into $\mathrm{x}$-rays through the SSC mechanism. Blandford (1990) gives the electromagnetic luminosity, $L_{\mathrm{em}}$, as

$$
L_{\mathrm{em}} \approx 10^{45} \mathscr{M}_{8}^{2}\left(\frac{a}{m}\right)^{2} B_{4}^{2} \mathrm{erg} \mathrm{s}^{-1},
$$

where $\mathscr{C}_{8}$ is the black hole mass in units of $10^{8} \mathscr{M}_{\odot}, B$ is the magnetic field in units of $10^{4} \mathrm{G}, a$ is the specific angular momentum, and $m=G M / c^{2}$. The x-ray luminosity in high luminosity radio-loud quasars could therefore be proportional to $L_{\mathrm{em}}$, say a fraction $g$ of $L_{\mathrm{em}}: L_{\mathrm{Xem}}=g L_{\mathrm{em}}$.

The luminosity due to the extraction of the gravitational energy of accreting gas, $L_{\text {accr }}$, in both radio-quiet and radioloud quasars has a different, linear, dependence on the black hole mass (Blandford 1990):

$$
L_{\mathrm{opt}} \approx L_{\mathrm{accr}} \sim 1.3 \times 10^{46} \mathscr{M}_{8} \frac{L_{\mathrm{accr}}}{L_{\mathrm{Edd}}} \mathrm{erg} \mathrm{s}^{-1} .
$$

We may identify $L_{\text {accr }}$ with the optical-UV luminosity, with the $\mathrm{x}$-ray luminosity in radio-quiet quasars, and with some part of the $x$-ray luminosity of radio-loud quasars, say a fraction $f$ of $L_{\text {accr }}: L_{\text {Xaccr }}=f L_{\text {accr }}$.

Comparing these two equations then defines a critical mass for radio-loud quasars at which $L_{\text {Xem }}$ equals $L_{\text {Xaccr }}$. This mass is

$$
\mathscr{C}_{8} \sim \frac{f}{g} 13 \frac{L_{\mathrm{accr}}}{L_{\mathrm{Edd}}}\left(\frac{a}{m}\right)^{-2} B_{4}^{-2} .
$$

We can now interpret the critical luminosity of $\log L_{\text {opt }} \sim 32-33 \mathrm{erg} \mathrm{s}^{-1} \mathrm{~Hz}^{-1}$ (Fig. 4) as corresponding to this critical mass for steep spectrum radio-loud quasars. For an Eddington limited source this corresponds to a black hole mass of $10^{9}-10^{10} \mathscr{C}_{\odot}$, since the luminosity per decade, $L_{\text {opt }}=10^{47}-10^{48} \mathrm{erg} \mathrm{s}^{-1}$.

Then,

$$
\frac{f}{g} \sim 0.077 \mathscr{M}_{8}\left(\frac{L_{\text {accr }}}{L_{\text {Edd }}}\right)^{-1}\left(\frac{a}{m}\right)^{2} B_{4}^{2} \sim 0.2-2,
$$

if $a / m=0.5$ and $L_{\text {accr }}=L_{\text {Edd }}$, i.e., $g$ is the order of $f$.

$f$ can be estimated from the $\alpha_{\text {ox }}$ measured in high $z$ radioquiet quasars. The observed $\alpha_{\text {ox }}$ of $\sim 1.8$ corresponds to a ratio of optical to $\mathrm{x}$-ray luminosity per decade of $\sim 100$, i.e., $f \sim 0.01$.

In this scenario the energy connected with the extraction of the black hole rotational energy is only inefficiently converted into $x$-ray photons, or into any photons at all. Thus, the bulk $(\sim 99 \%)$ of the power extracted from the spin of the black hole must go into kinetic energy of accelerated particles or be carried far away from the $\mathrm{x}$-ray emission region by the electromagnetic field.

Following Blandford (1990), the lifetime of a quasar powered only by the extraction of the spin of the black hole is:

$$
t_{\mathrm{em}, 8} \approx 16.7 \mathscr{M}_{8}^{-1} B_{4}^{-2},
$$


where $t_{8}$ is the time in units of $10^{8} \mathrm{yr}$. For the range of masses we considered before we have $t_{8}=0.2-2$. This is actually a lower limit, since the accreting gas could deposit angular momentum into the black hole and spin it up. The corresponding accretion time scale, or Eddington, time is: $t_{\mathrm{Edd}, 8} \approx 4$, so that $t_{\mathrm{em}, 8}<t_{\mathrm{Edd}, 8}$ for $\mathscr{C}_{8}>4$. In this scenario some of the high luminosity radio-quiet quasars could be objects which were powerful radio sources in the past, but whose central black hole has in the meantime completely slowed down.

\section{CONCLUSIONS}

In summary, ROSAT PSPC colors of 17 high-redshift quasars show that the $\mathrm{x}$-ray spectral properties of radio-loud and radio-quiet quasars at $z \approx 3$ have evolved differently: either only radio-loud quasars show absorption, or only radio-quiet quasars change their emitted power law spectrum. This implies different changes in the environment or emission mechanism of the two types of quasar. Distinguishing the two spectral possibilities should be straightforward with more high energy measurements.

Some of the data reported in this paper came from the ROSAT data bank. This work was supported by NASA Grants NAGW-2201 (LTSARP), NAG5-1872, NAG5 -1536, and NAG5-1680 (ROSAT), and NASA contracts NAS8-39073 (ASC) and NAS5-30934 (RSDC). This work was also supported by NSF Grants RII-8800660, INT9010583, and AST-9058510, and a gift from Sun Microsystems.

\section{REFERENCES}

Avni, Y., \& Tananbaum, H. 1986, ApJ, 305, 83

Bahcall, J., \& Peebles, J. 1969, ApJ, 156, L7

Baldwin, J. 1977, MNRAS, 178, 67P

Band, D. L., Cohen, R. D., Blanco, P. R., Junkkarinen, V. T., Burbidge, E. M., Rothschild, R. T., \& Reichert, G. A. 1994, in Multi-Wavelength Continuum Emission of AGN, edited by T. J.-L. Courvoisier and A. Blecha (Kluwer, Dordrecht), p. 318

Bechtold, J., et al. 1994, AJ, 108, 374 (Paper II)

Blandford, R. D. 1990, Active Galactic Nuclei, Saas-Fee Advanced Course

20, edited by T. J.-L. Courvoisier and M. Mayor (Springer, Berlin)

Blandford, R. D., \& Znajeck, R. L. 1977, MNRAS, 179, 433

Boyle, B. J., Fong, R., \& Shanks, T. 1987, MNRAS, 227, 717

Boyle, B. J., Griffiths, R. E., Shanks, T., Stewart, G. C., \& Georgantopoulos, I. 1993, MNRAS, 260, 49

Cavaliere, A., \& Padovani, P. 1988, ApJ, 315, 411

Chokshi, A., \& Turner, E. L. 1992, MNRAS, 259, 421

Elvis, M., Fiore, F., Wilkes, B. J., McDowell, J., \& Bechtold, J. 1994, ApJ, 422 (Feb. 10) (in press) (Paper I)

Fiore, F., Elvis, E., Siemiginowska, A., Wilkes, B. J., \& McDowell, J. C. 1994, ApJ (submitted)

Fink, H. H., \& Briel, U. G. 1993, A\&A, 274, L45

Haardt, F., \& Maraschi, L. 1993, ApJ, 413, 507

Haehnelt, M. G., \& Rees, M. J. 1993, MNRAS, 263, 168

Henry, P., et al. 1994, A. J., 107, 1270
Hoag, A. A., \& Smith, M. G. 1977, ApJ, 217, 362

Laor, A., Fiore, F., Elvis, M., Wilkes, B. J., \& McDowell, J. C. 1994, ApJ (submitted)

Molthagen, K., Wendker, H. J., \& Briel, U. G. 1994, A\&A (in press)

Morrison, R., \& McCammon, D. 1983, ApJ, 270, 119

Netzer, H. 1990, Active Galactic Nuclei, Saas-Fe Advanced Course 20, edited by T. J.-L. Courvoisier and M. Mayor (Springer, Berlin)

O’Dea, C. P., Baum, S. A., \& Stanghellini, C. 1991, ApJ, 380, 66

Osmer, P. 1977, ApJ, 214, 1

Padovani, P., \& Rafanelli, P. 1988, A\&A, 205, 53

Pearson, T. J., \& Readhead, A. C. S. 1984, in VLBI and Compact Radio Sources, IAU Symposium No. 110 (Reidel, Dordrecht), p. 15

Pfefferman, E., et al. 1987, Proc. SPIE, 733, 519

Pickering, T., \& Impey, C. 1994 (in preparation)

Small, T., \& Blandford, R. 1992, MNRAS, 259725

Tananbaum, H., et al. 1979, ApJ, 234, L9

Trümper, J. 1983, Adv. Space Res., 2, 241

Walter, R., \& Fink, H. H. 1993, A\&A, 274, 105

Wandel, A., \& Mushotzky, R. F. 1986, ApJ, 339, 674

Wilkes, B. J., \& Elvis, M. 1987, ApJ, 323, 243

Wilkes, B. J., Tananbaum, H., \& Worrall, D. M. 1994, ApJS, 92, 53

Williams, O. R., et al. 1992, ApJ, 389, 157

Worrall, D. M., Giommi, P., Tananbaum, H., \& Zamorani G. 1987, ApJ, 313, 596 